Author Accepted Manuscript of:

Clark DR, Lambert MI, Grigson C \& Hunter AM (2019) Increased strength is associated with lower trunk muscle activation during loaded back squats and dynamic body weight jumps. Translational Sports Medicine. https://doi.org/10.1002/tsm2.97.

This is the peer reviewed version of the following article: Clark DR, Lambert MI, Grigson C \& Hunter AM (2019) Increased strength is associated with lower trunk muscle activation during loaded back squats and dynamic body weight jumps. Translational Sports Medicine. DOI: 10.1002/tsm2.97, which has been published in final form at https://doi.org/10.1002/tsm2.97. This article may be used for non-commercial purposes in accordance with Wiley Terms and Conditions for Use of Self-Archived Versions. 
TRANSFERRED ORIGINAL ARTICLE

Running Author: CLARK et al

\title{
Increased strength is associated with lower trunk muscle activation during loaded back squats and dynamic body weight jumps
}

\author{
David R. Clark 1,2,
}

Email d.r.clark@ljmu.ac.uk

Michael I. Lambert ${ }^{3}$

Chris Grigson 2

Angus M. Hunter 2

AQ1

AQ2

1 School of Sport and Exercise Sciences, Faculty of Science, Liverpool John Moore's University, Liverpool, UK

2 Physiology, Exercise and Nutrition Research Group, Faculty of Health Sciences and Sport, University of Stirling, Stirling, UK

3 Division of Exercise Science and Sports Medicine, Department of Human

Biology, University of Cape Town, Cape Town, South Africa

\section{Correspondence}

David R. Clark, School of Sport and Exercise Sciences, Faculty of Science, Liverpool John Moore's University, Liverpool, UK.

Email: d.r.clark@ljmu.ac.uk

\section{Abstract}

This study measured how back squat strength (1RM) affected trunk muscle activation in performing squats, squat jump (SJ), and countermovement jump (CMJ). Fifty males, completed two test sessions. Squat 1RM was tested first. Participants were assigned to three groups: (a) strong group (SG), (b) middle group (MG), or (c) weak group (WG), based on relative squat 1 RM. Test 2: EMG data were collected for four trunk muscle sites; rectus abdominus, external oblique, lumbar sacral erector spinae, and upper lumbar erector spinae while performing (3 reps) SJ, CMJ, and squats at $65 \%, 75 \%$, and 95\% 1RM. Squat and jump phases were determined from a linear transducer and $30^{\circ}$ tertiles for each phase, from a knee goniometer. Normalized root mean square RMS increased significantly with load for each muscle site in both squat phases. Trunk muscle activation was significantly lower in SG vs WG in eccentric and concentric squat phases. Concentric and flight phase RMS in both jumps was lower in SG vs WG. RMS increased significantly for each eccentric tertile and first concentric tertile. Greater squat strength is associated with lower trunk muscle activation in squats and jumps and trunk muscle activation was highest in the two deepest $30^{\circ}$ squat segments. In conclusion, back squat strength training to parallel, where top of thighs are horizontal, is an effective method of developing dynamic trunk stability. 


\section{Keywords}

muscular strength; neuromuscular activation; surface EMG; trunk stability

\section{INTRODUCTION}

Several researchers have demonstrated effectiveness of loaded, compound exercises in activating trunk stabilizers.[1, 2, 3, 4, 5] Traditional core stability exercises, characterized by isolated isometric exercises do not reflect trunk control in dynamic human movement.[6, 7] Several studies reported effective trunk muscle activation for selected dynamic exercises,[8] squats,[1,2] standing, seated, unilateral and bilateral exercises,[9] and deadlifts.[3] Effects of specific exercise characteristics on trunk muscle activation have also been investigated, including instability,[5] external load,[1,2] trunk rotation,[10] and movement velocity.[10] Hence, there is good evidence that variations of loaded, dynamic, compound exercises, including back squat, are effective in activating trunk stabilizer muscles.[6,7] Despite this, barbell-loaded compound exercises, including back squat are not widely recognized as effective exercises for development of dynamic trunk stability.

We previously demonstrated acceptable reliability and sensitivity of sEMG in measuring trunk muscle activation in back squat.[1] We and others have shown a load effect for trunk muscle activation in this exercise.[1, 2, 3, 4, 11] Activation increased by load in eccentric and concentric phases for all four trunk muscle sites: rectus abdominus (RA), external oblique (EO), lumbar sacral erector spinae (LSES), and upper lumbar erector spinae (ULES).[1, 2] Higher trunk muscle activation in concentric phase of loaded back squat compared to the descent has been demonstrated in our laboratory[1,2] and by others.[11, 12] Understanding how trunk muscle activation responds to the same relative back squat load in strong compared to weaker participants will contribute to establishing this exercise as an effective method of developing dynamic trunk stability.

The impact of maximal strength and training status on lower limb muscle activation in the squat has been well reported. Quadriceps muscle activation in back squat 1RM test was significantly higher in trained compared to untrained subjects.[13] Higher activation in trained participants explained, in part, their capacity to lift higher 1RM loads and complete more reps in a single set to failure at 85\% 1RM.[13] Higher activation during maximal contractions in trained individuals is associated with increased motor unit recruitment, synchronization of motor unit discharge and improved intermuscular coordination.[13, 14] These neuromuscular measures of lower limb muscles therefore explain greater strength, power, and strength endurance performance associated with squat strength training status. Understanding trunk muscles adaptation to squat training and how these adaptations contribute to improved performance has significant relevance to applied strength training.

Hip adductor and quadriceps activation was higher in the final $30^{\circ}$ of descent and first $30^{\circ}$ of ascent than any other part of both phases of loaded parallel squats. $[15,16]$ Hence, for effective lower limb muscle activation, minimal optimal squat range is parallel, or where top of the thighs are horizontal. $[15,16]$ Prime movers are fundamental to squat performance, however equally important is the trunk's capacity to resist forward flexion and maintain trunk integrity while applying eccentric and concentric force through the full movement. Hence, quantifying changes in trunk muscle activation through full range of loaded back squat is important to inform training guidelines for effective development of dynamic trunk stability.

There is evidence that loaded squats have an acute potentiation effect on jumps[17, 18] and a chronic training effect on improving jump performance.[19, 20] The relationship between back squat strength and performance in countermovement jump (CMJ) and squat jumps (SJ) is well established.[17, 18, 19] However, despite the obvious importance of trunk 
stability in transferring force and power through the full kinetic chain, acute muscle activation has not been reported for SJ and CMJ. Hence, analysis of trunk muscle activation in CMJ and SJ would add to our understanding of these two commonly used exercises. Furthermore, it would explain the influence of back squat training on trunk muscle activation, and hence stability in CMJ and SJ performance.

The role of stabilizers, including trunk stabilizers in dynamic squat and jump performance has not been reported. However, back squat coaching guidelines include many cues to ensure that trunk stability and integrity is maintained under load through full range of movement. Similarly, in the applied setting the concept of "leaking" power or force from segments of the kinetic chain during performance of dynamic speed and power activities is well established. This relates to synchronized stabilization of the kinetic chain to optimize transfer of force and power generated by agonists in the direction of movement; this has received little scientific attention, especially for dynamic compound movements, possibly due to methodological limitations.

The aim of this study was to investigate the impact of back squat strength on trunk muscle activation in back squat at moderate to heavy loads. Secondary aims were to (a) analyze changes in trunk muscle activation through eccentric and concentric phases of the squat, and (b) investigate trunk muscle activation in SJ and CMJ and determine the effect of squat strength on trunk stabilization in these dynamic athletic actions.

\section{MATERIALS AND METHODS}

\subsection{Participants}

We recruited 50 participants with a mean back squat $1 \mathrm{RM}$ of $122 \mathrm{~kg}$ (SD $\pm 34 \mathrm{~kg}$ ), which translated to a relative 1RM (1RM/Body mass, $\mathrm{kg}$ ) of 1.5 (SD \pm 0.3 , Range: 0.8 to 2.2) (Table 1). Based on relative back squat $1 \mathrm{RM}$, participants were assigned to one of three groups for comparative analysis; Strong group (SG) $(n=16)$, Middle group (MG) $(n=17)$ and Weak group (WG) $(n=17)$. The three groups were significantly different according to absolute back squat $1 \mathrm{RM}\left(F_{2,47}=51.1, P<.0001\right)$ and relative back squat $1 \mathrm{RM}\left(F_{2,47}=125.7, P<.001\right)$. WG and SG were significantly different for strength training age $\left(F_{2,47}=3.6, P<.05\right)$, squat training age $\left(F_{2,47}=5.0, P<.05\right)$, SJ height $\left(F_{2,94}=8.8, P<.01\right)$, and CMJ height $\left(F_{2,94}=8.8\right)$, $P<.05)$. Body mass was significantly higher in SG compared to $M G\left(F_{2,47}=5.6, P<.01\right)$.

Table 1 Mean $( \pm S D)$ descriptive data for all participants (Total) and by group

\begin{tabular}{|c|c|c|c|c|}
\hline & $\begin{array}{l}\text { Total } \\
n-50\end{array}$ & $\begin{array}{l}\text { Weak } \\
\text { group } \\
\text { n-17 }\end{array}$ & $\begin{array}{l}\text { Middle } \\
\text { group } \\
\mathrm{n}-17\end{array}$ & $\begin{array}{l}\text { Strong group } \\
n-16\end{array}$ \\
\hline Age (y) & $22.3 \pm 3.1$ & $20.8 \pm 4.2$ & $22.4 \pm 4.1$ & $23.9 \pm 2.8$ \\
\hline Body mass (kg) & $81.5 \pm 11.2$ & $79.8 \pm 8.5$ & $76.7 \pm 11.1$ & $88.4 \pm 10.4^{* \star \star \star \star \star *}$ \\
\hline Strength training age $(\mathrm{y})$ & $5.3 \pm 3.1$ & $3.9 \pm 1.7$ & $5.5 \pm 3.5$ & $6.6 \pm 3.2^{* \pm}$ \\
\hline
\end{tabular}




\begin{tabular}{|c|c|c|c|c|}
\hline Squat training age (y) & $4.1 \pm 2.9$ & $2.6 \pm 1.1$ & $4.1 \pm 3.3$ & $5.6 \pm 3.0^{\text {*ะ }}$ \\
\hline Back squat 1RM (kg) & $122 \pm 34$ & $93 \pm 13$ & $114 \pm 19$ & 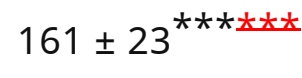 \\
\hline $\begin{array}{l}\text { Relative back squat } 1 \mathrm{RM} \\
\text { (ratio) }\end{array}$ & $1.5 \pm 0.3$ & $1.2 \pm 0.1$ & $1.5 \pm 0.1$ & $1.8 \pm 0.1^{* \star \star \star \star \star \star \star *}$ \\
\hline SJ height (cm) & $39.0 \pm 6.1$ & $36.6 \pm 4.6$ & $38.2 \pm 5.6$ & $42.7 \pm 6.4^{\text {* }}$ \\
\hline CMJ height (cm) & $40.9 \pm 6.1$ & $38.8 \pm 5.1$ & $40.2 \pm 6.1$ & $44.0 \pm 5.8^{\star \star 太}$ \\
\hline
\end{tabular}

Abbreviations: 1RM, 1 repetition maximum; CMJ, countermovement jump; MG, middle group; SG, strong group; SJ, squat jump; WG, weak group.

*Significant differences: WG vs SG $(P<.05)$,

**MG vs SG $(P<.01)$, and

***all groups $(P<.01)$.

Participants were recruited from a range of university and club sports including rugby, football, American football, tennis, and swimming. All participants were injury-free at the time of the study and had no history of injury with a bearing on back squat exercise. University of Stirling, School of Sport Research Ethics Committee granted ethical approval for the study, which was done in accordance with the Helsinki declaration (2013). All participants gave informed written consent prior to testing.

\subsection{Experimental design}

Participants attended the laboratory on two separate occasions with 3-7 days between visits. During the first visit, participants' training history was assessed, followed by the measurement of body mass, height, and back squat 1RM test. During the second visit, surface SEMG and kinematic data were measured for three repetitions of SJ, CMJ, and back squat at three progressive loads based on 1RM. Fixed testing order was used to avoid potentiation of the exercise tested second.[17] Both sessions began with a standardized warm-up and participants rested for 3 minutes between each test set. Testing was conducted at the same time of day to account for circadian rhythm.[21] Participants followed usual dietary intake and avoided strenuous exercises for 24 hours prior to testing.

\subsection{Back squat $1 \mathrm{RM}$ test}

The standardized warm-up before back squat 1RM testing comprised of a range of dynamic, compound body weight exercises that progressed to loaded barbell back squat for three sets of $10-8$ reps at $20 \mathrm{~kg}, 45 \%$ and $55 \%$ of estimated $1 \mathrm{RM}$. The standardized warm-up was repeated before the neuromuscular test protocol and on both occasions' technical requirements of SJ, CMJ, and back squat depth were rehearsed and confirmed.

The back squat $1 \mathrm{RM}$ and neuromuscular test protocols were conducted in a safety squat rack (FT700 Power Cage, Fitness Technology) using competition approved barbell and disks (Eleiko). All squat efforts were assessed for correct technique and depth by primary investigator, an experienced strength coach. The 1RM test protocol, previously described by McGuigan (2016),[22] was used to determine maximal load completed with correct technique and depth within five attempts. Participants were required to descend to where top of their thighs were horizontal or lower followed by a continuous ascent to full extension of hips and knees. Participants were instructed to control descent cadence and drive the bar up as fast as possible, with control, during ascent.[23] Participants had a three-minute seated rest between each test repetition. 
Back squat training and test loads calculated according to system mass max (SM) assume that $89 \%$ of body mass is included in external load.[1, 2] The remaining $11 \%$, (ie, shanks and feet) do not move vertically in squat exercise.

- $\mathrm{SM}=1 \mathrm{RM}+(0.89 \times$ body mass $)(\mathrm{kg})$

- External load $=(S M \times$ percentage of $S M)-(0.89 \times$ body mass $)(\mathrm{kg})$.

This method is established in back squat research[1,2] and was applied to calculate back squat warm-up and test loads in this study.

\subsection{Kinematic data}

A single linear transducer (Celesco, PT5A), fitted to the safety squat cage directly above the participant was attached to the middle of the barbell for back squat and a wooden dowel for jump tests. The linear transducer measured displacement and time from initiation of the descent, transition from descent to ascent to end of ascent for each rep of back squat and jump tests. This facilitated identification of eccentric and concentric phases and jump height for SJ and CMJ tests.

An electro mechanical goniometer, incorporating a high precision rotary potentiometer (6657s-1-103; Bourns), was attached to right knee to measure flexion and extension. The fixed rotary potentiometer of the goniometer was placed at the center of rotation of the knee. The fixed arm of the goniometer was attached to the lateral thigh by surgical tape. The actuating goniometer arm was attached to the lateral calf by Velcro onto a neoprene sleeve, reinforced with surgical tape. The actuating arm incorporated three hinges to allow natural extension through the movement and a compact swiveling gimbal to accommodate small angular movement. The goniometer was manually calibrated with a fixed plastic protractor.[24]

A threshold was established within Acqknowledge software (version 4.4.2; Biopac Systems Inc) to produce a digital output to indicate correct knee flexion had been reached corresponding to required back squat depth. Correct execution of technique resulted in an audible sound. When the sound was not heard, the repetition was excluded and repeated. Minimum knee flexion that corresponded to back squat depth, where thighs were horizontal or lower, was established for each participant during the first warm-up set with the barbell.

Linear transducer data synchronized to root mean square (RMS) data were used to demarcate eccentric and concentric phases in back squat and jump tests and flight phase in jump tests. Back squat goniometer data were used to segment eccentric and concentric phases into three equal $30^{\circ}$ (degree) tertiles for RMS analysis.[15]

\subsection{Neuromuscular test}

On arrival, participants were re-screened for injury or illness and weighed to update calculation of system mass max and test loads. Electrodes were fixed to four trunk muscle sites. Muscle sites were shaved, abraded, and cleaned with an alcohol swab.[25] At each muscle site, two adhesive electrodes (Ambu WhiteSensor WS, Ambu) were attached longitudinally along muscle fiber orientation with a $20 \mathrm{~mm}$ inter-electrode space according to Surface EMG (sEMG) for Non-Invasive Assessment of Muscles (SENIAM) guidelines.[25] Electrodes from two muscle sites were connected to a BioNomadix 2 Ch. EMG Wireless Transmitter (BN-EMG2). Two wireless transmitters were secured in a harness: one on the upper back for posterior muscle sites; lumbar sacral erector spinae (LSES) and upper lumbar erector spinae (ULES), and the second for rectus abdominus (RA) and external oblique (EO) on the mid-chest. Cables and transmitters were secured to minimize artifact noise and 
prevent interference with execution of the exercises. Standardized warm-up was completed up to and including SJ and CMJ rehearsal, thereafter participants were prepared for neuromuscular and kinematic data capture.

Wireless transmitters were attached, and goniometer fixed to the right knee. Transmitters were matched with a receiver unit in a Biopac MP150 system (Biopac Systems Inc) which facilitated transmission of high resolution sEMG signals at a rate of $2000 \mathrm{~Hz}$. Three back squat warm-up sets followed: 10 reps at $20 \mathrm{~kg}$ (barbell), 10 reps at 45\% SM, 8 reps at 55\% SM. Neuromuscular and kinematic data signals were checked and any issues were rectified during these sets. Minimum squat depth was determined during 10 reps at $20 \mathrm{~kg}$ and programmed into the Biopac system via the knee goniometer.

The barbell was replaced by a wooden dowel $(<0.25 \mathrm{~kg})$ and attached to the linear transducer for SJ and CMJ tests. In both jump tests, participants were instructed to reset completely between each rep, perform maximal concentric efforts and ensure that the dowel remained in contact with the shoulders throughout. In SJ, participants began by squatting to parallel, where thighs were horizontal and paused for a count of 3 before jumping. Squat depth in CMJ was self-selected and followed immediately by a maximal jump effort.[26] Participants had a three-minute seated rest between each test set.

The wooden dowel was replaced with $20 \mathrm{~kg}$ barbell (Eleiko) and back squat tests were conducted for three reps at 65\%,75\%, and 95\% SM with 3 minutes rest between each set. Back squat test loads were calculated using system mass max formula, incorporating individual 1RM score and body mass measured at start of the neuromuscular test session.

\subsection{Surface electromyography}

Trunk muscle sEMG was measured from four sites on right-hand side of the body; RA, EO, LSES, and ULES. Bilateral symmetry has been established for these muscles.[27] Reliability of SEMG analysis of trunk muscle activation at these sites has been demonstrated in our laboratory[1,2] and by others.[4, 28]

Surface EMG was sampled at a rate of $2000 \mathrm{~Hz}$ and anti-aliased with a $500 \mathrm{~Hz}$ low pass filter in the Biopac MP150 system. The resulting signals were processed by applying an averaged RMS filter with a rolling 100-ms wide Bartlett window. Once processed, mean RMS was extracted for eccentric and concentric phase using the synchronized linear transducer data, and for each tertile using synchronized knee goniometer signal. Mean RMS for three reps of all test sets in back squat and jump tests were normalized to mean concentric phase RMS at $65 \%$ SM.

Previous work from our laboratory demonstrated that submaximal, dynamic RMS normalization methods were more reliable and sensitive than normalizing to maximal isometric RMS methods in the back squat.[1,2,29] Dynamic, submaximal normalization of lower limb muscle EMG was found to be more effective than isometric maximal voluntary contraction (iMVC) method in measuring activation in dynamic activities including, back squat,[29] cycling,[30] and running.[31] Furthermore: (a) Dankaerts et al[32] reported greater within and between day reliability for dynamic submaximal EMG normalization than iMVC for trunk muscles in healthy and back pain patients; and (b) Burden (2010) showed that iMVC force was dependent on training status and suggested that EMG generated in this way resulted in an inconsistent denominator for normalization.[33] Based on this evidence and the dynamic nature of our tests, we selected to normalize all RMS to mean concentric back squat RMS at $65 \%$ SM.

\subsection{Data analysis}


Muscle activation data are presented as mean normalized RMS percentage $( \pm S D)$. RMS data for four muscle sites in back squat and jumps (SJ \& CMJ) were analyzed and presented separately for the whole cohort. RMS data were combined for all group and tertile analyses.

Back squat neuromuscular test data were analyzed and presented for concentric and eccentric phase and by tertile for each phase.[15] Knee goniometer data were used to divide eccentric and concentric phases into three equal $30^{\circ}$ segments or tertiles for RMS analysis.[15] Tertiles are referred to as E-1, E-2, E-3, C-1, C-2, C-3, where E-1 is first tertile of the descent, C-1 first tertile of ascent and C-3, final tertile leading back to full extension.

RMS data for the jumps are presented for three phases: (a) eccentric; (b) concentric; and (c) flight. The concentric RMS sample was limited to the segment of the phase where participants were in contact with the ground and able to apply downward force. The end of the concentric phase was identified from linear transducer data as concentric or upward displacement point that corresponded with upright standing position determined at start of eccentric phase. The flight phase was from this point, upright standing, to the point where peak displacement was reached. As a result, flight phase included plantar flexion or the segment from flat foot, upright standing to toe off. According to Linthorne[34], this segment of kinematic jump analysis using a force platform would be included in the concentric phase. However, a recent study suggests that the primary role of plantar flexors in jump performance is to stabilize the knee joint in early concentric phases.[35] They conclude that plantar flexion at the end of explosive concentric knee and hip extension, makes little or no contribution to jump performance.

\subsection{Statistical analysis}

All statistical analyses were performed on GraphPad Prism software (version 7.00). Normal distribution of relative back squat 1 RM data was established using D'Agostino \& Pearson normality test $(\alpha=.05)$. Using back squat RMS (ULES) from 75\%-95\% SM, we performed post hoc sample size calculation using G*Power model for three independent groups at $90 \%$ power. Using G*Power one-way ANOVA for 3 independent groups (weak, medium, and strong), a priori sample size compute based on $\alpha .05$ at .95 power and Cohen's effect size of 1.87 from mean ULES concentric RMS at $75 \%(117.3+17)$ vs. $95 \%(196.6+57)$ necessitates a minimal sample size of 9 participants per group. We exceed this number thereby avoiding type I and II errors.

A two-way analysis of variance (ANOVA) was used to determine the differences in participant data (back squat $1 \mathrm{RM}, \mathrm{S}$, and $\mathrm{CMJ}$ height and training history) and trunk muscle activation (normalized RMS percentage) measures between groups and test conditions. Significant load and group effect were followed by Tukey's post hoc procedure for multiple comparisons. Level of significance of $(P<.05)$ was selected to determine statistical differences.

\section{RESULTS}

\subsection{Neuromuscular data}

Neuromuscular data analyzed for eccentric and concentric phase of back squat for all participants, three test loads and four muscle sites are reported in Table 2A,B. Mean RMS percentage increased significantly in response to load in eccentric $\left(F_{2,588}=47.9, P<.0001\right)$ and concentric phase $\left(F_{2,588}=225.3, P<.0001\right)$ with an interaction effect for contraction type across loads (squat phase $\times$ load) $\left(F_{2,588}=3.9, P<.001\right)$. Activation increased in all muscle sites for each load increment (65\%-75\% SM and 75\%-95\% SM) for both squat phases and this was significant in 17 out of 24 instances. Mean RMS percentage increased from $65 \%$ 
to $95 \%$ SM for each muscle site in eccentric phase by: RA- $27 \%$, EO-31\%, LSES- $47 \%$ and ULES$44 \%$ and concentric phase by: RA-56\%, EO-83\%, LSES-64\%, and ULES-97\%.

Table 2 Difference in mean percentage RMS between test loads for each muscle site in (A) eccentric and (B) concentric phase. Difference in eccentric and concentric mean percentage RMS for each test load and muscle site $(C)$. All participants $(n=50)$

\section{A. Eccentric}

\begin{tabular}{|c|c|c|c|c|c|c|}
\hline \multirow{2}{*}{\multicolumn{2}{|c|}{$\frac{\text { Test loads }}{65 \%}$}} & \multirow[b]{2}{*}{$75 \%$} & \multirow[b]{2}{*}{$95 \%$} & \multicolumn{2}{|c|}{ Mean $\Delta$ (\% RMS) } & \multirow[b]{2}{*}{$95 \%-65$} \\
\hline & & & & $75 \%-65 \%$ & & \\
\hline RA & 62.7 & 71.2 & 89.5 & -8.5 & ns & $-26.8^{*}$ \\
\hline EO & 98.5 & 111.9 & 129.1 & -13.5 & ns & $-30.6^{*}$ \\
\hline LSES & 83.4 & 97.8 & 130.8 & -14.4 & ns & $-47.4^{*}$ \\
\hline ULES & 96.6 & 104.7 & 141.0 & -8.0 & ns & $-44.3^{*}$ \\
\hline \multicolumn{7}{|c|}{ B. Concentric } \\
\hline & \multicolumn{3}{|l|}{ Test loads } & \multicolumn{3}{|c|}{ Mean $\Delta(\%$ RMS $)$} \\
\hline & $65 \%$ & $75 \%$ & $95 \%$ & $75 \%-65 \%$ & $95 \%-65 \%$ & $95 \%-75$ \\
\hline RA & 100 & 111.5 & 156.2 & $-11.5 \mathrm{~ns}$ & 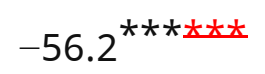 & $-44.7^{*}$ \\
\hline EO & 100 & 120.6 & 183.1 & $-20.6^{\star \star}$ & $-83.1^{\star \star \star \star \star \star \star \star ~}$ & $-62.6^{*}$ \\
\hline LSES & 100 & 115.6 & 161.4 & $-15.6^{\star \star 太}$ & $-61.4^{* \star \star \star \star \star \star *}$ & $-45.7^{*}$ \\
\hline ULES & 100 & 117.4 & 196.7 & $-17.4^{\star \star \star \star}$ & $-96.7^{\star \star \star \star \star \star \star \star *}$ & $-79.3^{*}$ \\
\hline \multicolumn{7}{|c|}{ C. Eccentric vs Concentric } \\
\hline & \multicolumn{3}{|c|}{ Mean $\Delta$ (\% RMS) } & & & \\
\hline & $65 \%$ & $75 \%$ & $95 \%$ & & & \\
\hline RA & $-37.4^{* \star \star \star \star \star \star \star \star \star *}$ & $-40.3^{* \star \star \star \star \star \star \star \star *}$ & $-66.8^{* \star * \star \star \star \star *}$ & & & \\
\hline EO & $-1.5 \mathrm{~ns}$ & $-8.6 \mathrm{~ns}$ & $-54.1^{\star \star \star \star \star \star \star *}$ & & & \\
\hline LSES & $-16.6 \mathrm{~ns}$ & $-17.8^{\star \star}$ & $-30.6^{* \star \star \star \star \star \star *}$ & & & \\
\hline ULES & $-3.4 \mathrm{~ns}$ & $-12.7 \mathrm{~ns}$ & $-55.7^{\star \star \star \star \star \star \star \star ~}$ & & & \\
\hline
\end{tabular}

Abbreviations: EO, external oblique; LSES, lumbar sacral erector spinae; ns, not significantly different; RA, rectus abdominus; RMS, root mean squared (EMG); ULES, upper lumbar erector spinae. 
*Significant differences: $(P<.05)$,

$* *(P<.001)$, and

$* * *(P<.0001)$.

\subsection{Back squat: eccentric/ concentric}

\subsubsection{All participants}

Concentric RMS was significantly $\left(F_{7,343}=28.8, P<.0001\right)$ greater than eccentric RMS in the following muscle sites and loads; RA-65\% SM (37\%), 75\% SM (40\%), and 95\% SM (67\%), EO95\% SM (54\%), LSES-75\% SM (18\%) and 95\% SM (31\%) and ULES-95\% SM (56\%) (Table 2C). There was an interaction effect for RMS (muscle site $\times$ load) in the concentric phase $\left(F_{6}\right.$, $588=3.9, P<.001)$ but not the eccentric phase $\left(F_{2,588}=47.9, P<.0001\right)$.

\subsubsection{Group analysis}

Combined mean RMS data for all four trunk muscle sites revealed that activation in SG was significantly lower than WG for all test loads in eccentric phase $\left(F_{2,141}=26.7, P<.05\right)$ and at $95 \% \mathrm{SM}$ in concentric phase $\left(F_{2,141}=26.7, P<.05\right)$ (Figure 1$)$. Strong group RMS was significantly lower than MG at 95\% SM in the eccentric phase. 


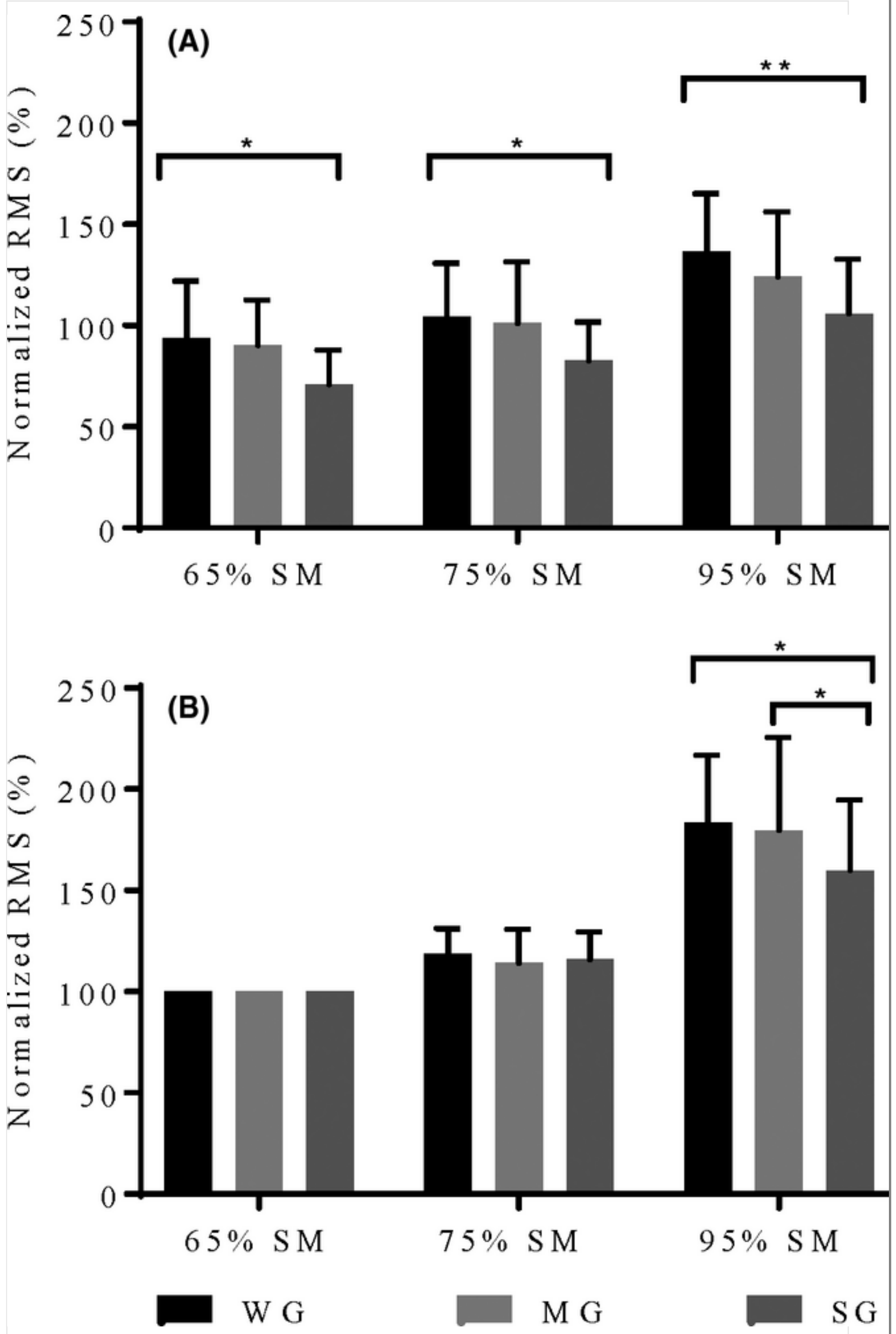

Fig. 1 
Normalized back squat mean RMS percentage for three groups (WG, MG and SG), three test loads; $65 \%, 75 \%$, and $95 \%$ SM in the; A, Eccentric phase and B, Concentric phase. Significant differences: $* P<.05, * \star P<.01$. (Abbreviations: MG, middle group; RMS, root mean squared; SG, strong group; SM, system mass max; $\mathrm{SM}$, system mass max; WG, weak group)

\subsection{Back squat: tertiles}

\subsubsection{All participants}

There was a significant load effect $\left(F_{2,98}=209.8, P<.0001\right)$ and interaction $\left(F_{10,490}=29.3\right.$, $P<.0001)$ by tertile across the three test loads. RMS increased significantly $\left(F_{5,245}=77.2\right.$, $P<.0001)$ for each descending tertile (E1, E2, and E3) of the squat and peaked during the first part of the upward movement (Tertile C1) for each test load (Figure 2). RMS in the final two concentric tertiles (C2 and C3) decreased as ascent progressed to full extension of hips and knees.

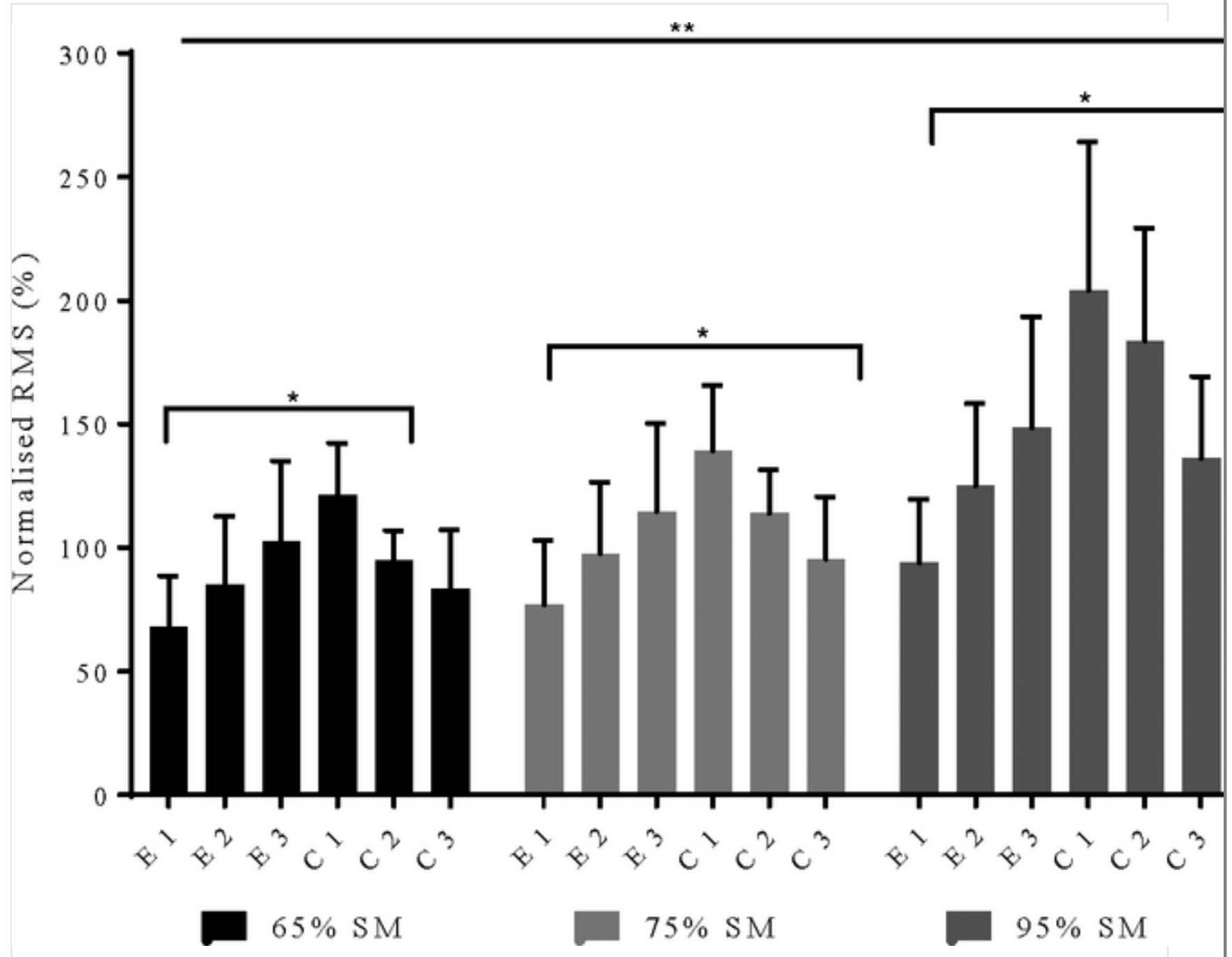

Fig. 2 Normalized back squat mean RMS percentage for all participants, three tertiles for each phase, eccentric and concentric and three test loads; $65 \%, 75 \%$, and 95\% SM. *Significant differences between tertiles within each load, $P<.0001$, **Significant load effect $P<.0001$. (Abbreviations: C, concentric; E, eccentric; RMS, root mean squared; SM, system mass max) 


\subsubsection{Group analysis}

RMS was lower in SG compared to WG in all three eccentric tertiles for all loads apart from tertile $\mathrm{E}-1$ at $95 \% \mathrm{SM}\left(F_{5,235}=35.6, P<.0001\right)$ (Figure 3). In tertile C-1 at 95\% SM, both the WG and MG had higher activation than SG $\left(F_{2,47}=3.6, P<.01\right)$. There were no differences between groups in concentric tertiles at $65 \%$ and $75 \%$ SM and for C-2 and C-3 at 95\% SM.
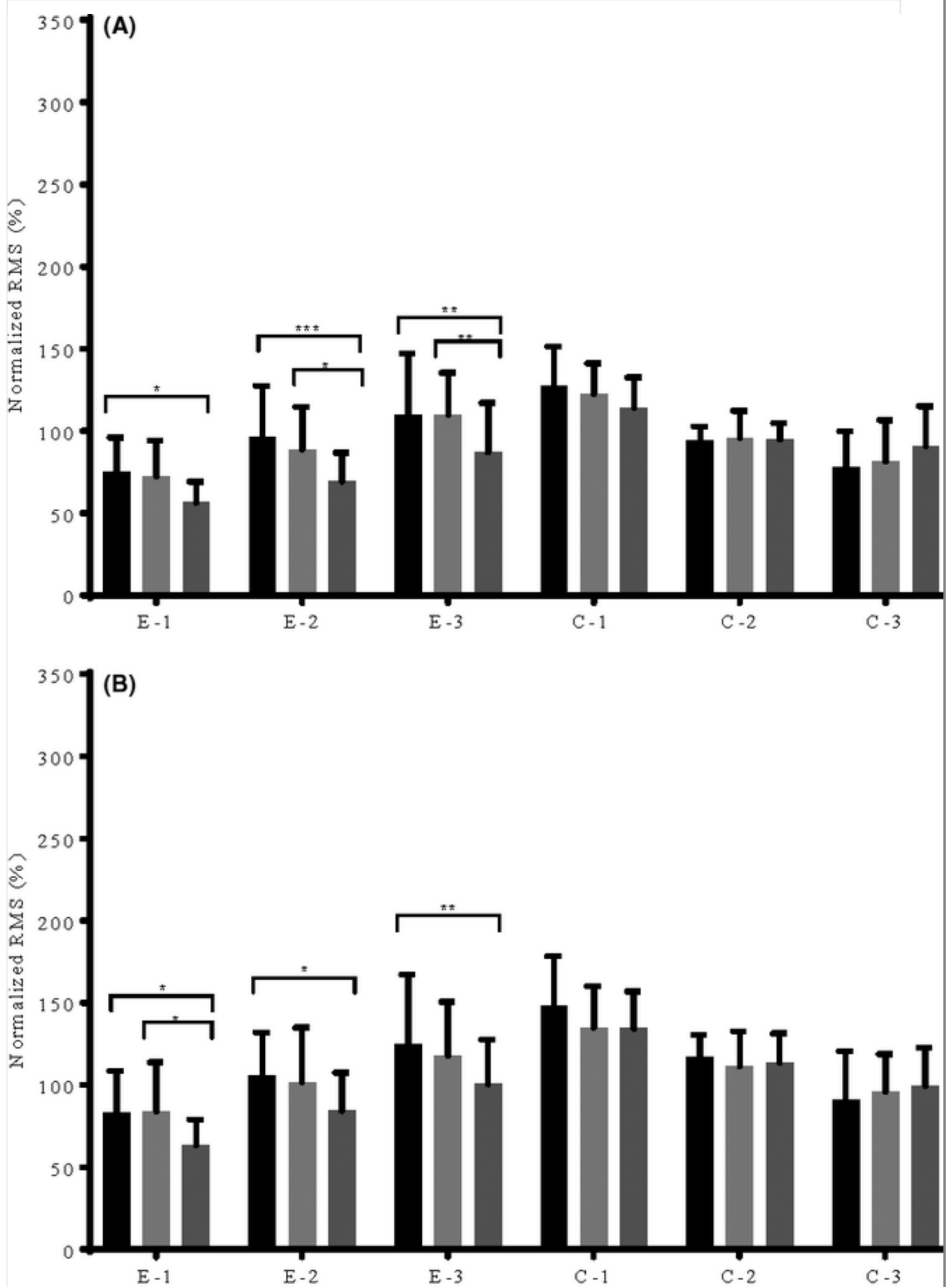


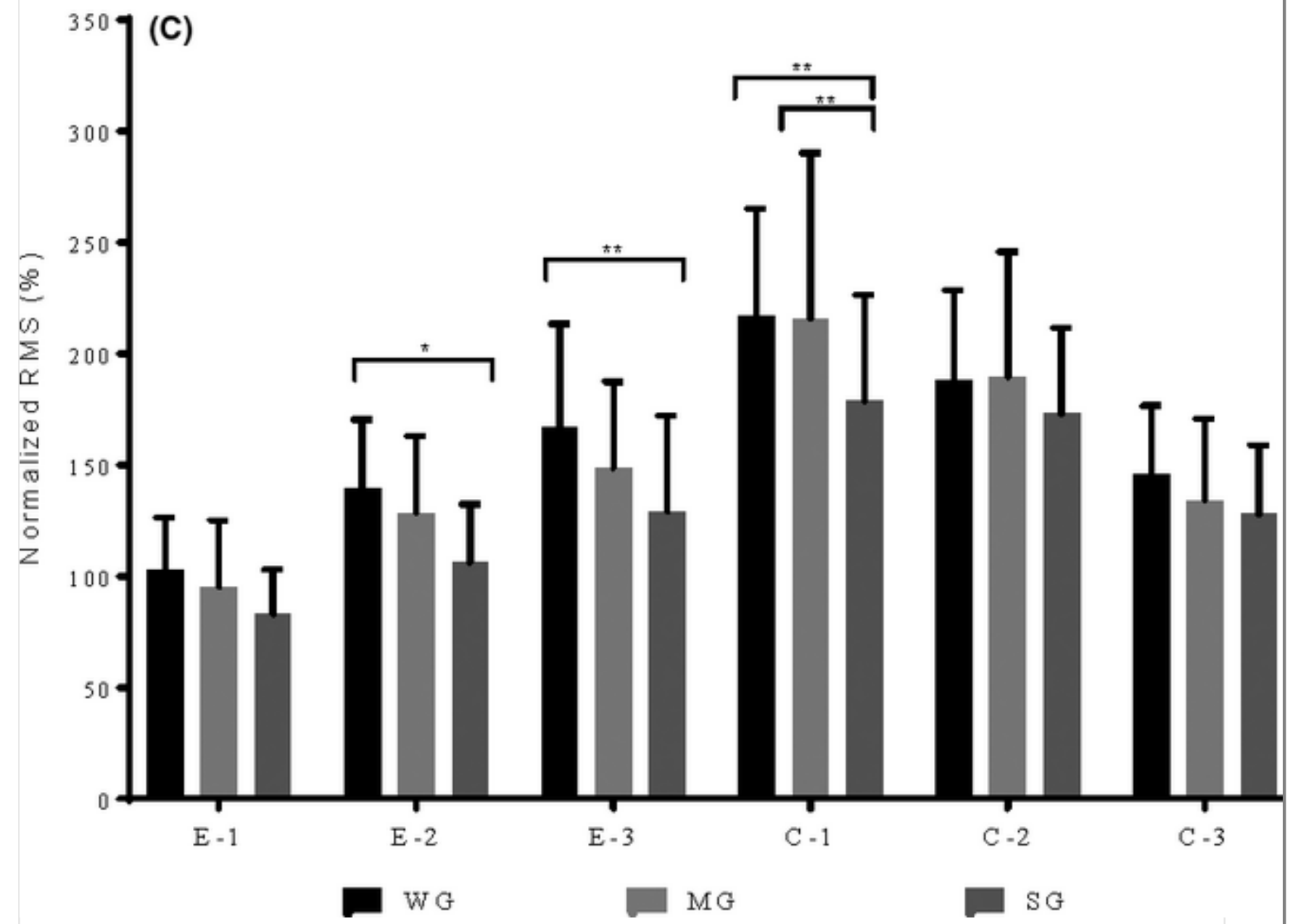

Fig. 3 Normalized back squat mean RMS percentage for three groups in three tertiles for eccentric and concentric phases. Test loads: A, 65\% SM, B, 75\% SM, and C, $95 \%$ SM. Significant differences: $* P<.05, * * P<.01, * \star * P<.001$. (Abbreviations: $C$, concentric; $E$, eccentric; MG, middle group; RMS, root mean squared; SG, strong group; SM, system mass max; WG, weak group)

\subsection{Squat and countermovement jump}

\subsubsection{All participants}

RMS was significantly higher in concentric and flight phase compared to eccentric phase for both jumps in RA and EO $\left(F_{2,300}=75.8, P<.0001\right)$ (Table 3$)$. LSES activation was significantly higher in the concentric phase compared to eccentric in SJ $(84 \%, P<.05)$ and CMJ $(92 \%$, $P<.05)$. RA activation in flight phase of SJ was significantly higher than in concentric phase $(73 \%, P<.91)$.

Table 3 Mean percentage RMS for each muscle site and jump phase in (A) Squat jump and (B) Countermovement jump for all participants $(n=50)$

A. Squat jump 


\begin{tabular}{|c|c|c|c|c|c|c|}
\hline RA & 58.9 & 301.4 & 374.8 & $-242.4^{* \star \star \star \star \star \star \star}$ & $-315.9^{* \star * \star \star \star \star *}$ & $-73.4^{\star \star}$ \\
\hline EO & 143.8 & 277.5 & 281.5 & $-133.7^{\star \star \star \star \star \star \star \star *}$ & $-137.8^{* \star \star \star \star \star *}$ & $-4.1 \mathrm{~ns}$ \\
\hline LSES & 40.3 & 124.4 & 87.6 & $-84.1^{\star \star}$ & $-47.3 \mathrm{~ns}$ & $36.8 \mathrm{~ns}$ \\
\hline ULES & 68.3 & 130.5 & 128.1 & $-62.2 \mathrm{~ns}$ & $-59.8 \mathrm{~ns}$ & $2.4 \mathrm{~ns}$ \\
\hline \multicolumn{7}{|c|}{ B. Countermovement jump } \\
\hline & \multicolumn{3}{|c|}{ Mean \% RMS } & \multicolumn{3}{|l|}{ Mean $\Delta \%$ RMS } \\
\hline & Eccentric & Concentric & Flight & Ecc vs Con & Ecc vs FT & Con vs FT \\
\hline RA & 94.9 & 319.4 & 369.7 & 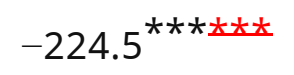 & $-274.9^{\star \star \star \star \star \star \star *}$ & $-50.4 \mathrm{~ns}$ \\
\hline EO & 192.2 & 348.5 & 299.6 & 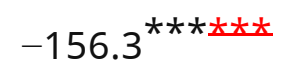 & $-107.4^{* \star \star \star \star}$ & $48.9 \mathrm{~ns}$ \\
\hline LSES & 53.6 & 145.3 & 94.7 & $-91.7^{\star \star}$ & $-41.1 \mathrm{~ns}$ & $50.6 \mathrm{~ns}$ \\
\hline ULES & 95.6 & 158.2 & 133.0 & $-62.6 \mathrm{~ns}$ & $-37.4 \mathrm{~ns}$ & $25.2 \mathrm{~ns}$ \\
\hline
\end{tabular}

Abbreviations: Con, concentric phase; Ecc, eccentric phase; EO, external oblique; FT, flight phase; LSES, lumbar sacral erector spinae; ns, not significant; RA, rectus abdominus; RMS, root mead squared (EMG); ULES, upper lumbar erector spinae.

*Significant differences: $(P<.05)$,

$* *(P<.001)$, and

$* * *(P<.0001)$.

In the eccentric phase, EO activation was significantly greater than RA and LSES in CMJ and all other sites in SJ (Figure 4). RA and EO RMS was significantly greater than LSES and ULES activation in concentric and flight phase in both SJ $\left(F_{3,588}=49.0, P<.0001\right)$ and $\mathrm{CMJ}\left(F_{3}\right.$, $588=51.5, P<.0001)$ (Figure 4).
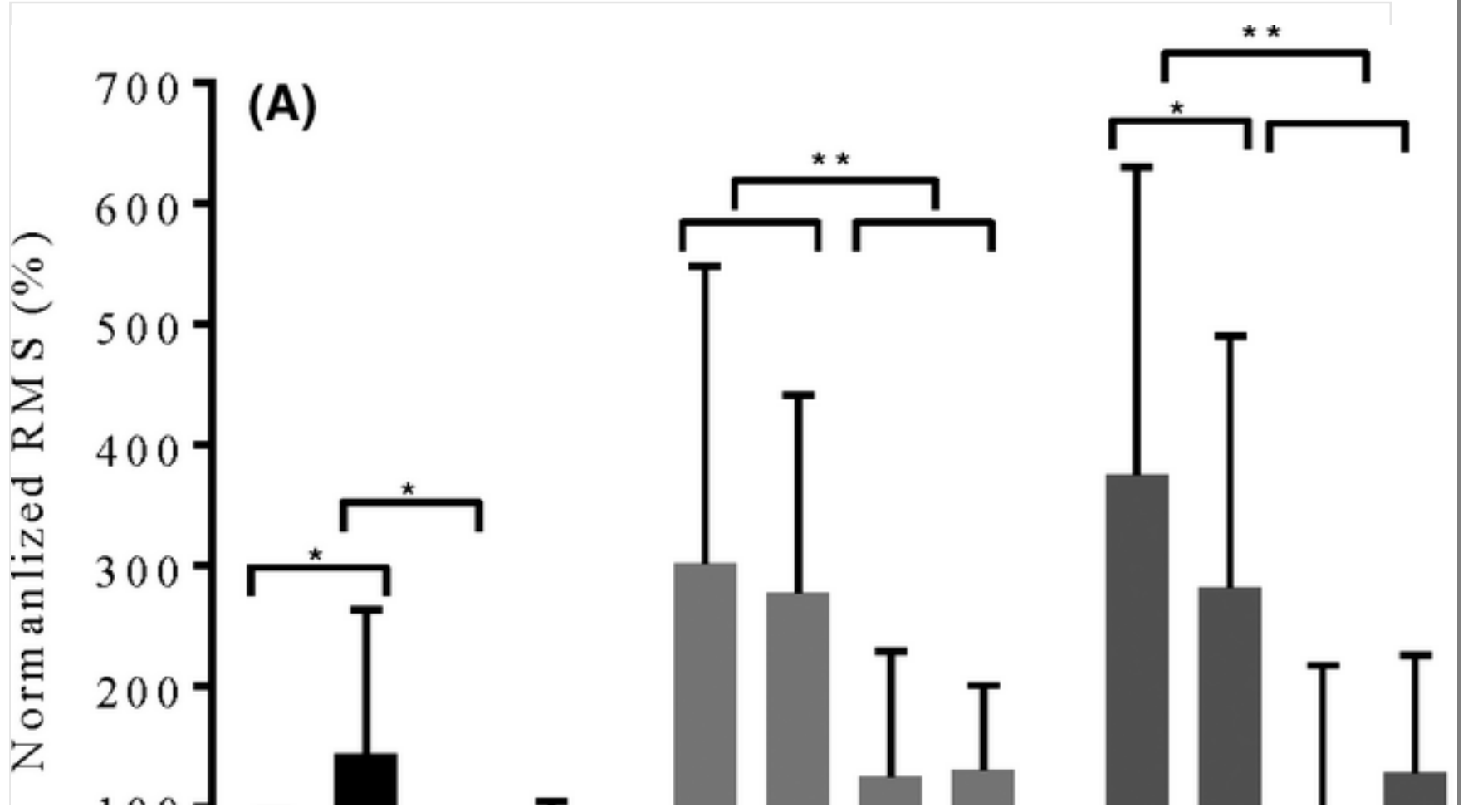


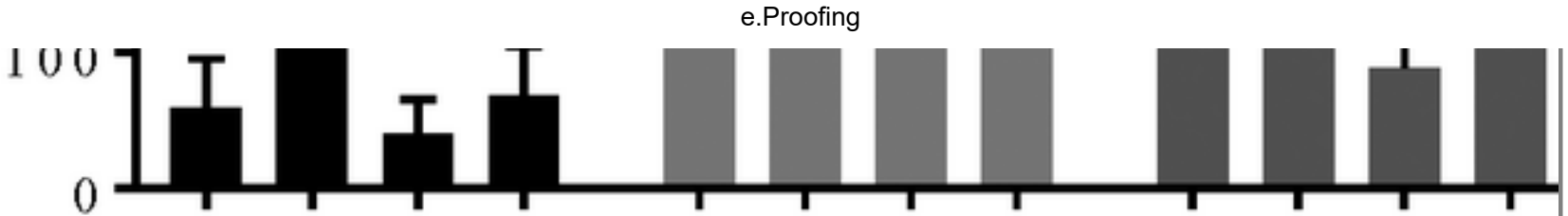

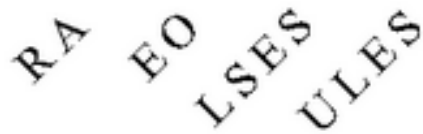

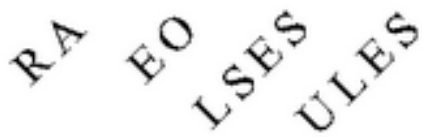

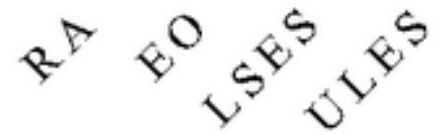

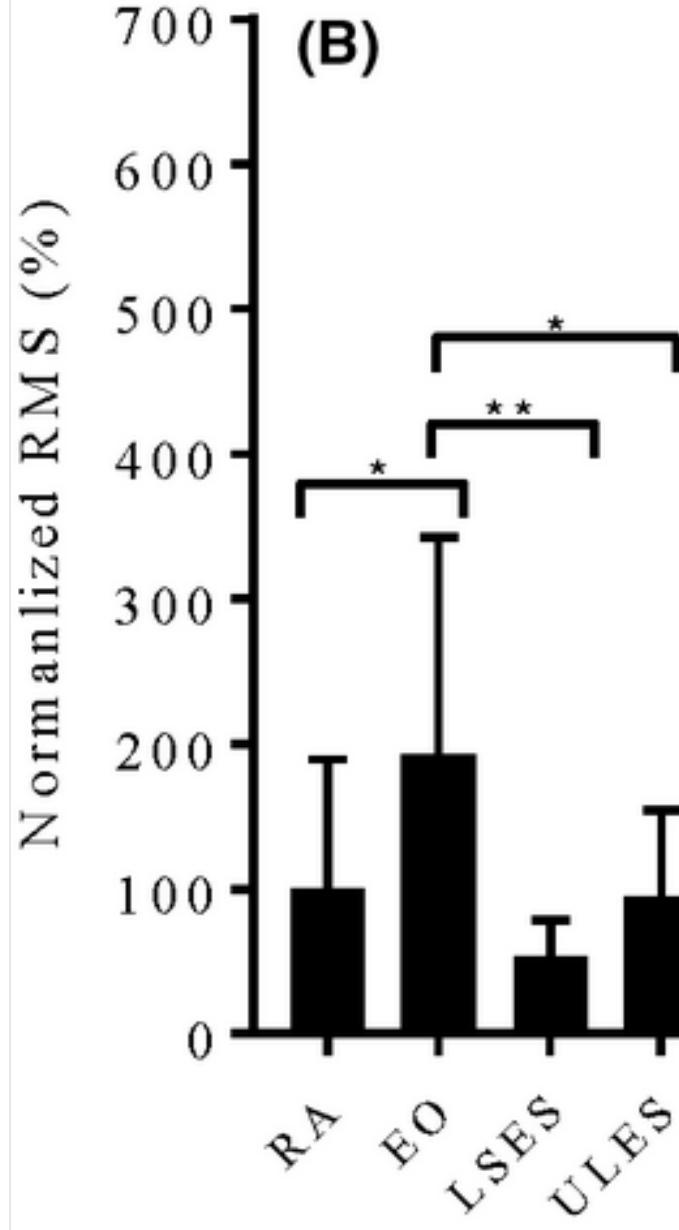

Eccentric
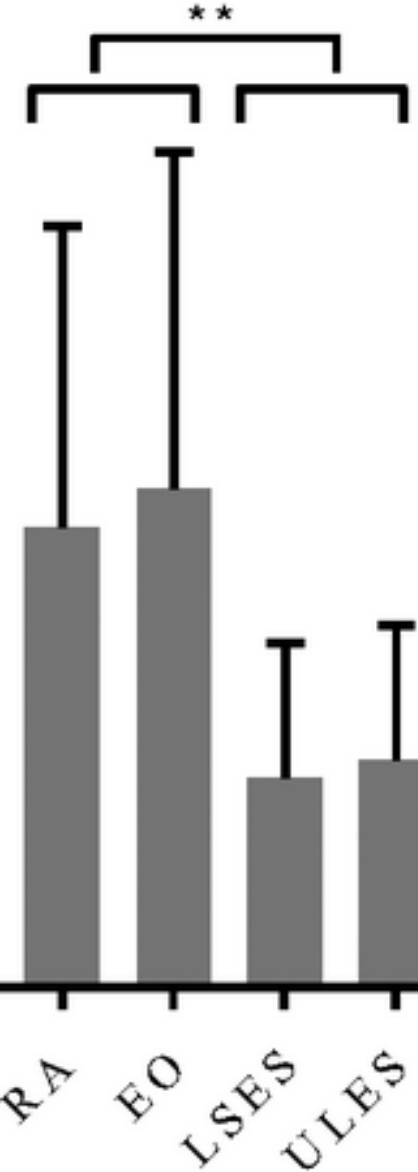

Concentric

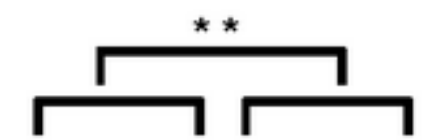

Fig. 4 Normalized back squat mean RMS percentage all participants by muscle site in eccentric, concentric and flight phase of, $A$, squat jump and $B$, countermovement jump. Significant differences $* P<.05, * * P<.01$. (Abbreviations: EO, external oblique; LSES, lumbar sacral erector spinae; RA, rectus abdominus; RMS, root mean squared; ULES, upper lumbar erector spinae)

\subsubsection{Group analysis}

In RMS analysis of combined muscle sites, activation in concentric and flight phase of both jumps was significantly lower in SG compared to WG $\left(F_{2,144}=54.5, P<.05\right)$ (Figure 5). SG activation was lower than $M G$ in concentric phase of SJ $\left(F_{2,144}=54.5, P<.05\right)$. 


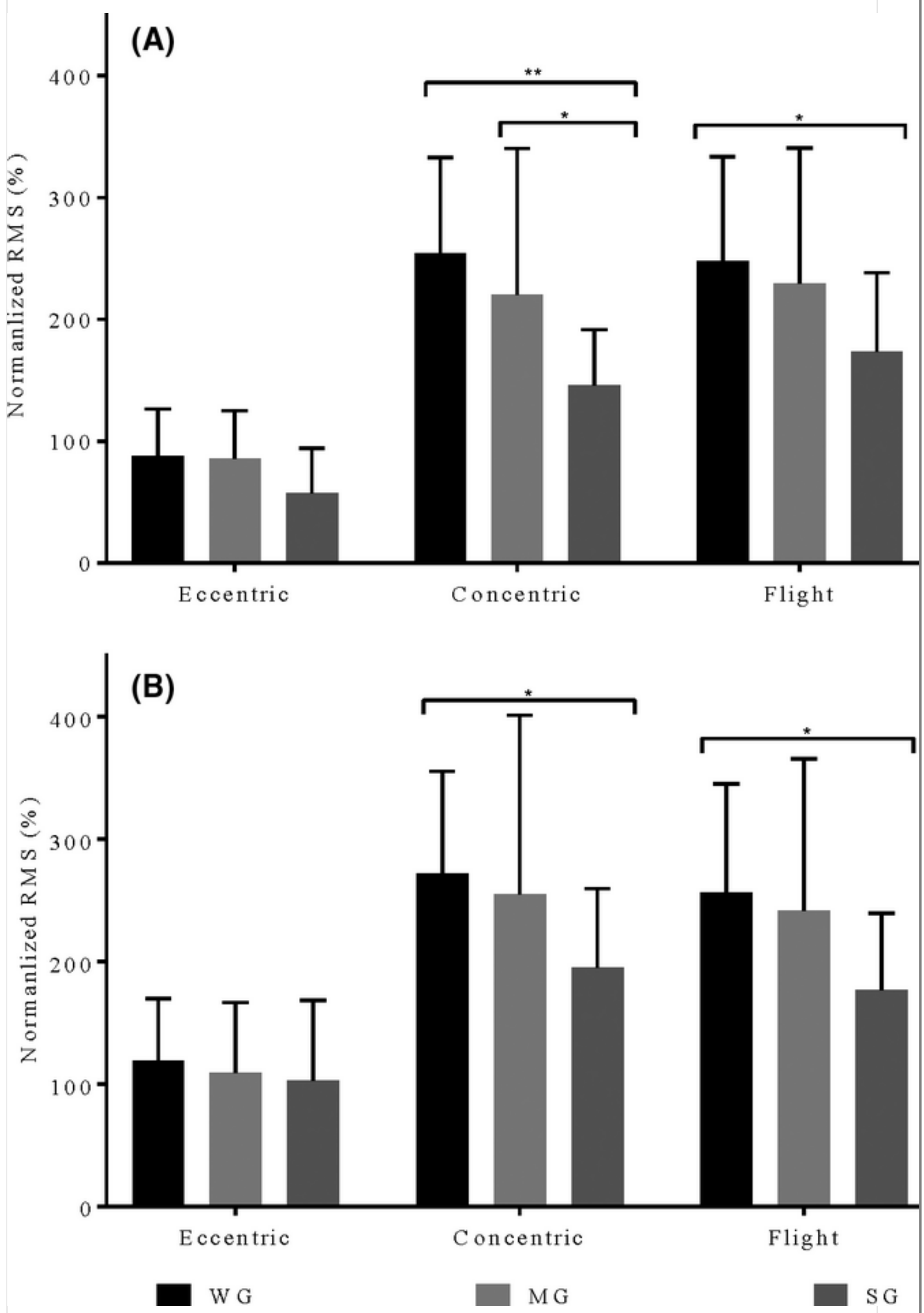

Fig. 5 Normalized SJ and CMJ mean RMS percentage for three groups in eccentric, concentric, and flight phase; $A$, squat jump and B, countermovement jump. 
Significant difference between groups $* P<.05, * \star P<.001$. (Abbreviations: MG, middle group; RMS, root mean squared; SG, strong group; WG, weak group)

\section{DISCUSSION}

This study measured the impact of back squat strength on trunk muscle activation in back squat, squat jump and countermovement jump. High levels of back squat strength were associated with lower trunk muscle activation in squats at moderate and heavy loads and in squat and countermovement jumps. Trunk muscle activation was highest in the lowest segments $\left(30^{\circ}\right.$ tertile) of descent and ascent of the parallel squat for all test loads. These novel findings contribute to a better understanding of loaded back squat training for improved performance and health outcomes.

\subsection{Back squat strength and trunk muscle activation}

Higher back squat strength results in greater lower limb agonist EMG response to maximal strength effort.[13,14] However, we found that higher levels of back squat strength resulted in lower levels of trunk muscle activation in eccentric and concentric back squat at moderate (75\% SM) and high loads (95\% SM) for all muscle sites (Table 2). Lower trunk muscle activation in SG compared to WG occurred at the same relative, but higher absolute back squat loads. Significant mean differences in test loads between WG and SG ranged from $40 \mathrm{~kg}(65 \% \mathrm{SM})$ to $64 \mathrm{~kg}(95 \% \mathrm{SM})$.

This finding provides new evidence that increases in back squat strength from regular, progressive squat training is achieved, in part through development of effective and efficient trunk stabilization. Hence, adaptations to stabilizers, in conjunction with agonists, $[13,14]$ underpin improvements in dynamic squat strength and associated performance gains. It is established that loaded squats are an effective method of activating trunk stabilizers, $[1,2]$ however, this is the first time effects of acquired squat strength on these muscles has been reported.

\subsection{Back squat tertile analysis: Trunk muscle activation}

Tertile analysis of RMS by group confirms and expands this finding. Trunk muscle activation was significantly lower in SG than WG for all eccentric tertiles and loads and first concentric tertile at 95\% SM (Figure 3). Therefore, squatting to parallel or deeper is most effective in activating the trunk stabilizers and squat strength training increases the capacity to stabilize the trunk more efficiently in the most challenging squat phases at higher absolute loads. This, along with evidence that lower limb muscle activation is highest in the deepest part of the eccentric and concentric phase of the parallel squat, $[13,14]$ provides additional and compelling support for squat training to this depth.

\subsection{Trunk muscle activation: Squat and countermovement jump}

Higher levels of back squat strength translated to lower trunk muscle activation in concentric and flight phase of SJ and CMJ. The SG, with significantly higher mean jump heights in SJ (17\%) and CMJ (13\%) had significantly lower trunk muscle activation than WG in these two phases. This demonstrates transfer of adaptation from a high load, low-velocity compound exercise, back squat, to a dynamic and power-oriented movement, central to performance in many sports and athletics activities. This suggests that improved efficiency in dynamic trunk stabilization mechanisms from heavy squat training may transfer positively to other dynamic athletic activities.

This is the first study to report trunk muscle activation in SJ and CMJ. For both jumps, concentric and flight phase trunk muscle activation was higher than eccentric activation. 
Mean normalized RMS percentage in concentric and flight phase was approximately $200 \%$ indicating high demand for trunk stabilization during these two phases. Particularly interesting is the high activation of trunk stabilizers during flight phase, where there is no ground contact and therefore direct force application.

Improved jump performance resulting from back squat training is possibly due to biomechanical similarity in body position of the squat exercise and jump tests.[36] Consequently, neurological and possibly morphological adaptations to squat training result in trunk muscle strength, which increases dynamic trunk stability. This results in greater transfer of agonist induced ground reaction force and therefore jump height.[36] In a previous study, we found that free barbell squat resulted in higher trunk muscle activation than the supported hack squat.[2] This was required to control unsupported external load through the full kinetic chain. The current study confirms that trunk muscle adaptation to free barbell back squat training facilitates greater resistance and transfer of force and power in ground based concentric and flight phases of explosive, dynamic jumps.

\subsection{Translation of findings}

Relative back squat $1 \mathrm{RM}$ of greater than $170 \%$ body mass achieved through progressive loaded squat training results in lower activation of trunk stabilizers in subsequent moderate to heavy squats compared to participants with a relative squat $1 \mathrm{RM}$ of $<140 \%$ of body mass. In the applied setting, the most important early adaptation to progressive loaded back squat training is primarily development of the trunk's capacity to maintain the position of the external load over the center of the base of support in the sagittal plane. Myer et al (2014) propose that tension developed by posterior, anterior and lateral abdominal muscles is fundamental to the maintenance of an upright posture throughout the entire squat movement.[37] Our study, therefore provides neuromuscular evidence underpinning this adaptation and this important and established applied squat coaching cue.

Secondly, the most relevant finding for athletic performance is that adaptation to progressive loaded back squat training translates directly to performance in dynamic body weight jumps. Neuromuscular adaptations to back squat training are associated with lower trunk muscle activation and higher jump height. Arguably, greater trunk stability and tension in dynamic jumps, facilitates more effective resistance and transfer of force generated by lower limb extension in concentric jump phase. Flight phase trunk muscle activation is similar to that in concentric phase, highlighting importance of trunk stability for effective kinetic control while not in ground contact. This explains in part, the greater ground reaction force and higher jump heights in squat training studies.[20,36] This study presents novel neuromuscular evidence to support the proposal that squat strength develops trunk stability and that this contributes to improved jump performance.

The third key finding with applied relevance is impact of squat depth on activation of trunk stabilizers. Similar to findings for lower limb agonists,[15] trunk muscle activation in this study peaked in the lowest segments of squat descent and ascent. Furthermore, parallel squats are associated with greater jump performance, previously thought to result exclusively from improved agonist induced rate of force development.[38] Our data provides new evidence suggesting that the role of the squat exercise in improved jump performance may be due to enhanced dynamic trunk stability, while compounding the justification for squatting to parallel.

\section{PERSPECTIVE}

This is the first study to show that back squat strength training adaptations reduce trunk muscle activation in squat jump, countermovement jump and moderate to heavy squats. Trunk muscle activation in back squat is highest in the last third of squat descent and first 
third of ascent, confirming importance of parallel squats to develop optimal trunk stability. Trunk muscle activation in these demanding segments of parallel back squat was significantly lower in trained participants compared to those less trained. Hence, loaded parallel barbell back squat is an effective training method for development of efficient trunk stability. Furthermore, trunk stability adaptations from back squat training contribute to improved dynamic jump performance.

These findings highlight possible future research areas including; (a) the impact of squat training adaptation on trunk muscle activation, stability and whole body performance in acceleration, sprint and change of direction speed, and (b) impact of squat strength, acquired through chronic training, on acute fatigue of the trunk stabilizers in dynamic athletic activities.

\section{ACKNOWLEDGEMENTS}

The authors would like to thank all those who participated in the study; athletes and colleagues from Sportscotland Institute of Sport, University of Stirling student athletes and club athletes from across Scotland.

\section{REFERENCES}

1 Clark D, Lambert MI, Hunter AM. Reliability of trunk muscle electromyography in the loaded back squat exercise. Int J Sports Med. 2016;37(6):448-456.

2 Clark DR, Lambert MI, Hunter AM. Trunk muscle activation in the back and hack squat at the same relative loads. Journal of Strength and Conditioning Research. 2019;33(Suppl 1):S60-S69.

3 Nuzzo JL, McCaulley GO, Cormie P, Cavill MJ, McBride JM. Trunk Muscle Activity During Stability Ball and Free Weight Exercises. J Strength Cond Res. 2008;22(1):95102.

4 Hamlyn N, Behm DG, Young WB. Trunk muscle activation during dynamic weighttraining exercises and isometric instability activities. J Strength Cond Res. 2007;21(4):1108-1112.

5 McBride JM, Larkin TR, Dayne AM, Haines TL, Kirby TJ. Effect of absolute and relative loading on muscle activity during stable and unstable squatting. Int J Sports Physiol Perform. 2010;5(2):177-183.

6 Wirth K, Hartmann H, Mickel C, Szilvas E, Keiner M, Sander A. Core Stability in Athletes: A Critical Analysis of Current Guidelines. Sport Med. 2016;47(3):401-414. https://doi.org/10.1007/s40279-016-0597-7.

7 Martuscello JM, Nuzzo JL, Ashley CD, Campbell BI, Orriola JJ, Mayer JM. Systematic review of core muscle activity during physical fitness exercises. J Strength Cond Res. 2013;27:1684-1698. https://doi.org/10.1519/JSC.0b013e318291b8da.

8 Comfort P, Pearson SJ, Mather D. An electromyographical comparison of trunk muscle activity during isometric trunk and dynamic strengthening exercises. J Strength Cond Res. 2011;25(1):149-154.

9 Saeterbakken AH, Fimland MS. Muscle activity of the core during bilateral, unilateral, seated and standing resistance exercise. Eur J Appl Physiol. 
2012;112(5):1671-1678. https://doi.org/10.1007/s00421-011-2141-7.

10 Watkins RG, Uppal GS, Perry J, Pink M, Dinsay JM. Dynamic electromyographic analysis of trunk musculature in professional golfers. Am J Sports Med. 1996;24(4):535-538. https://doi.org/10.1177/036354659602400420.

11 Aspe RR, Swinton PA. Electromyographic and Kinetic Comparison of the Back Squat and Overhead Squat. J Strength Cond Res. 2014;28:2828-2836. http://www.ncbi.nlm.nih.gov/pubmed/24662228

12 Gullett JC, Tillman MD, Gutierrez GM, Chow JW. A Biomechanical Comparison of Back and Front Squats in Healthy Trained Individuals. J Strength Cond Res. 2009;23(1):284-292.

13 Pick J, Becque DM. The relationship between training status and intensity on muscle activation and relative submaximal lifting capacity during the back squat. $J$ Strength Cond Res. 2000;14(2):175-181.

14 Aagaard P, Simonsen EB, Andersen JL, Magnusson P, Dyhre-Poulsen P. Increased rate of force development and neural drive of human skeletal muscle following resistance training. J Appl Physiol. 2002;93(4):1318-1326.

https://doi.org/10.1152/japplphysiol.00283.2002.

15 Pereira GR, Leporace G, Chagas DV, Furtado LF, Praxedes J, Batista LA. Influence of hip external rotation on hip adductor and rectus femoris myoelectric activity during a dynamic parallel squat. J Strength Cond Res. 2010;24(10):2749-2754

16 Yavuz HU, Erdağ D, Amca AM, Aritan S. Kinematic and EMG activities during front and back squat variations in maximum loads. J Sports Sci. 2015;33(10):1058-1066.

17 Mitchell CJ, Sale DG. Enhancement of jump performance after a 5-RM squat is associated with postactivation potentiation. Eur J Appl Physiol. 2011;111(8):19571963. https://doi.org/10.1007/s00421-010-1823-x.

18 Esformes J, Bampouras T. Effects of back squat depth on lower-body postactivation potentiation. J Strength Cond Res. 2013;27(11):2997-3000.

19 Wisloff U, Helgerud J, Hoff J. Strength and endurance of elite soccer players. Med Sci Sports Exerc. 1998;30(3):462-467.

20 Cormie P, McGuigan MR, Newton RU. Influence of strength on magnitude and mechanisms of adaptation to power training. Med Sci Sports Exerc. 2010;42(8):15661581. https://doi.org/10.1249/MSS.0b013e3181cf818d.

21 Atkinson G, Reilly T. Circadian variation in sports performance. Sports Med. 1996;21(4):292-312. http://www.ncbi.nlm.nih.gov/pubmed/8726347 Accessed October 9, 2014.

22 Haff GG, Triplett NT. Essentials of Strength Training and Conditioning. Vol 4 edition. 4th ed. (Haff GG, Triplett NT. eds.). Champaign, IL: Human Kinetics; 2016. doi:10.1016/S0031-9406(05)66120-2.

23 Cormie P, McBride JM, McCaulley GO. Validation of power measurement techniques in dynamic lower body resistance exercises. J App/ Biomech. 
24 Brandon R, Howatson G, Hunter A. Reliability of a combined biomechanical and surface electromyographical analysis system during dynamic barbell squat exercise. J Sports Sci. 2011;29(13):1389-1397.

25 Hermens HJ, Freriks B, Disselhorst-Klug C, Rau G. Development of recommendations for SEMG sensors and sensor placement procedures. J Electromyogr Kinesiol. 2000;10(5):361-374. https://doi.org/10.1016/S10506411(00)00027-4.

26 Suchomel TJ, Sole CJ, Stone MH. Comparison of methods that assess lower-body stretch-shortening cycle utilization. J Strength Cond Res. 2016;30(2):547-554. https://doi.org/10.1519/JSC.0000000000001100.

27 Vakos JP, Nitz AJ, Threlkeld AJ, Shapiro R, Horn T. Electromyographic activity of selected trunk and hip muscles during a squat lift. Effect of varying the lumbar posture. Spine (Phila Pa 1976). 1994;19(6):687-695.

http://www.ncbi.nlm.nih.gov/pubmed/8009334. Accessed February 22, 2015.

28 Anderson K, Behm DG. Trunk Muscle Activity Increases With Unstable Squat Movements. Can J Appl Physiol. 2005;30(1):33-45.

29 Balshaw TG, Hunter AM. Evaluation of electromyography normalisation methods for the back squat. J Electromyogr Kinesiol. 2012;22(2):308-319.

30 Albertus-Kajee Y, Tucker R, Derman W, Lambert MI. Alternative methods of normalising EMG during cycling. J Electromyogr Kinesiol. 2010;20(4):1036-1043. https://doi.org/10.1016/j.jelekin.2011.03.009.

31 Albertus-Kajee Y, Tucker R, Derman W, Lamberts RP, Lambert MI. Alternative methods of normalising EMG during running. J Electromyogr Kinesiol. 2011;21(4):579-586.

32 Dankaerts W, O'Sullivan PB, Burnett AF, Straker LM, Danneels LA. Reliability of EMG measurements for trunk muscles during maximal and sub-maximal voluntary isometric contractions in healthy controls and CLBP patients. J Electromyogr Kinesiol. 2004;14(3):333-342.

33 Burden A. How should we normalize electromyograms obtained from healthy participants? What we have learned from over 25years of research. J Electromyogr Kinesiol. 2010;20(6):1023-1035.

34 Linthorne NP. Analysis of standing vertical jumps using a force platform. $A m$ J Physiol. 2001;69(11):1198-1204. https://doi.org/10.1119/1.1397460.

35 Farris DJ, Lichtwark GA, Brown N, Cresswell AG. The role of human ankle plantar flexor muscle-tendon interaction and architecture in maximal vertical jumping examined in vivo. J Exp Biol. 2016;219(4):528-534.

https://doi.org/10.1242/jeb.126854.

36 Wirth K, Keiner M, Hartmann H, Sander A, Mickel C. Effect of 8 weeks of freeweight and machine-based strength training on strength and power performance. $J$ Hum Kinet. 2016;53(1):201-210. https://doi.org/10.1515/hukin-2016-0023. 
37 Myer GD, Kushner AM, Brent JL, et al. The back squat: A proposed assessment of functional deficits and technical factors that limit performance. Strength Cond J. 2014;36(6):4-27. https://doi.org/10.1519/SSC.0000000000000103.

38 Hartmann H, Wirth K, Klusemann M, Dalic J, Matuschek C, Schmidtbleicher D. Influence of Squatting Depth on Jumping Performance. J Strength Cond Res. 2012;26(12):3243-3261. https://doi.org/10.1519/JSC.0b013e31824ede62. 